CHAPTER 13

\title{
AUTOMATIC SLIDE SCANNING
}

\author{
JOSÉ L. PECH-PACHECO AND GABRIEL CRISTÓBAL
}

\begin{abstract}
In this chapter we describe an automatic slide scanning and autofocusing system that consists of three components. First, images are captured at low magnification. This yields a panoramic view of an entire slide which allows the extraction of the positions and sizes of all particles. Then, a particle screening is carried out at medium magnification, in order to eliminate particles that are not diatom-like, i.e. debris that does not comply with pre-defined selection criteria. This method selects about $80 \%$ of all usable valves. In a third step at high magnification, images of particles, mostly diatoms, are captured using autofocusing and multifocus fusion. The output of the system is an annotated gallery of images that can be used for diatom identification. The main limitation of the automatic slide scanning system consists of the mechanical parts. The average time to position the motor-controlled microscope stage and to acquire an image is about 3 seconds.
\end{abstract}

\section{Introduction}

Although some studies have explored the analysis of images at medium magnification, they have not considered the problem at the next level down, i.e. starting the automation process from a mosaic of low magnification images. This is a very challenging problem, because a diatom sample is normally contaminated with debris, broken diatom valves and valves which lie at an angle. We distinguish between two broad classes of diatom identification systems: semi-automatic and automatic. Semi-automatic methods require human interaction to select specimens from a low resolution image. Obviously, this provides good results but it is still time consuming, tedious and demands a lot of concentration. Methods for diatom detection and identification have been studied by Cairns et al. $(1972,1979)$ and Pech-Pacheco and Alvarez-Borrego (1998). Cairns et al. (1972) have proposed diatom identification methods based on coherent optics and holography. However, they did not consider the problem of automation with low resolution images. Culverhouse et al. (1996) described methods for identifying dinoflagellates, using neural networks, but again they did not provide a fully automatic method. Pech-Pacheco and Alvarez-Borrego (1998) proposed a hybrid optical-digital method for the identification of five different species of phytoplankton, using operators invariant to translation, rotation and scale. In Pech-Pacheco et al. (1999) correlation techniques are used for identifying phytoplankton specimens.

At low magnification it is difficult, if not impossible, to discriminate between diatom and non-diatom particles. Therefore, it is necessary to detect all possible "candidate" particles through image binarization on the basis of thresholding. Several methods have been proposed in the literature for threshold determination. 
Here, we will propose a simple but efficient method based on a modified histogram thresholding. Another key aspect in the automation process is a reliable and fast autofocusing method. Groen et al. (1985) have identified and compared 11 different autofocus algorithms. Different focusing criteria have been proposed (Krotkov, 1987; Vollath, 1987; Subbarao et al., 1993; Yeo et al., 1993; Nayar and Nakagawa, 1994; Bocker et al., 1996; Neveu, 1999). Most of them extract a focus measure that is maximum for the best-focused image. Autofocus algorithms can be classified into two categories: those based on the statistical variance of pixel values, and others based on the spatial frequency content of the image. Here, we propose an adaptive thresholding method that provides a good compromise between detectability and noise rejection. Also, a new autofocusing algorithm based on the computation of the variance of the image gradient, and some new focus metrics are proposed and compared with existing methods. We also propose the use of multifocus fusion techniques for combining in-focus details from different focal planes into a single image, in order to obtain both a sharp valve contour and striation pattern.

The overall procedure of the automatic slide scanning system consists of three steps. First, image acquisition at low magnification is used to obtain a panoramic view of the whole slide, which allows the extraction of the position and size of all particles. Second, an intermediate resolution particle screening is carried out in order to eliminate non-diatom particles. Third, images are captured at high magnification using autofocusing and multifocus fusion. The output of the system is an annotated gallery of images that can be used for diatom identification.

This chapter is organized as follows. The next section provides a general description of the equipment and data used. Section 3 presents the basis of automatic slide scanning in brightfield microscopy. Autofocusing methods are described in Section 4, whereas multifocus fusion techniques are described in Section 5. Section 6 contains concluding remarks.

\section{Material and methods}

Diatom samples were analyzed using a Zeiss Axiophot photomicroscope, with a $100 \mathrm{~W}$ halogen light source, and with $4 \mathrm{x}, 10 \mathrm{x}$ (low magnification), 20x (medium magnification) and 40x (high magnification) lenses. The ocular magnification was 0.6x. For image acquisition, we used the LG-3 grayscale frame grabber from Scion that includes the Scion Image software for Windows ${ }^{a}$, which is a version of the popular Macintosh program NIH Image. Scion Image allows several image processing operations, like capture, display, analyze, enhance, measure, annotate and output images. The LG-3 is connected to a Cohu 4910 series monochrome CCD camera, which is a high quality, economical choice for grayscale scientific imaging applications. The frame grabber resolution (CCIR) is $768 \times 512$ pixels and the pixel depth 8 bits. Two PCs (Pentium II and III) running Windows98 were used, the one for image acquisition and the other for computations. Furthermore, for time intensive calculations a SUN Enterprise 450 with four processors was used. The microscope stage was controlled with a H101 (4" + 3") controller from Prior Scientific, with a step size of $0.1 \mu \mathrm{m}$ for the $\mathrm{X}-\mathrm{Y}$ axes and $1 \mu \mathrm{m}$ for the $\mathrm{Z}$ axis.

${ }^{a}$ The Scion Imaging software can be downloaded free from http://www.scioncorp.com 
The total travel distance of the microscope stage for the $\mathrm{X}-\mathrm{Y}$-Z axes is $11 \times 7 \times 1$ $\mathrm{cm}$, respectively. Figure 1 shows a diagram of the system. The size range of the particles analyzed was between 20 and $260 \mu \mathrm{m}$, which can be considered part of the microplankton. Smaller diatoms (nanoplankton) cannot be observed using the available system, because they require oil-immersion lenses. The size ranges of the particles that can be observed with the lenses are as follows: 10x: range $=131-260$ $\mu \mathrm{m} ; 20 \mathrm{x}$ : $61-130 \mu \mathrm{m} ; 40 \mathrm{x}: 20-60 \mu \mathrm{m}$. In the future we intend to apply our methods to nanoplankton.

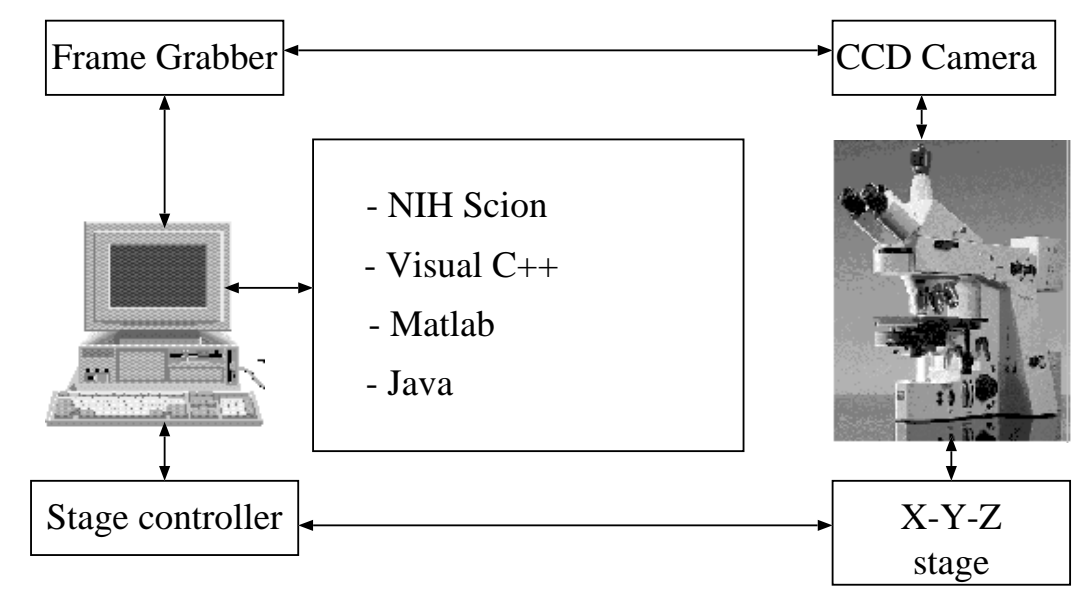

Figure 1. System configuration for automatic slide scanning. A brightfield microscope with a motorized stage attached is controlled by a PC. A CCD camera with frame grabber allows image acquisition.

\section{Automatic slide scanning}

\subsection{Automatic localization: overall procedure}

The overall procedure consists of two parts: image acquisition at low magnification and a further analysis at higher magnification. At low magnification the goal is to obtain a panoramic view of the entire slide by tiling images. The subsequent processing steps will provide the position and size of each particle, for a further analysis at high magnification. Figure 2 shows a schematic overview of the processing stages. Figure 2 (left) corresponds to the low magnification stage and Fig. 2 (right) corresponds to the higher magnification stage. Because the particles may appear at different depths, the localization method has been improved by combining different multifocus images, which allows the detection of particles that otherwise might be skipped (see Figs 9 and 10). Once the particles have been detected by applying image processing techniques (see below), we extract their positions and sizes. The size information allows us to select the correct lens for displaying the specimens at higher magnification. At high magnification (e.g. 40x) we apply the autofocusing, 
the particle screening and the multifocus fusion stages, which are described in the next sections. The output of the automatic system is a gallery of annotated images, some of which do not contain diatom valves, and which can be analyzed using the methods described in the other chapters of this book. We note again that at this stage of processing some of the images may contain debris. The goal of the particle screening is to remove a substantial number of particles (debris, broken valves) that are not required to be analyzed. Figure 3 shows two annotated particles from an image gallery provided by the automatic system.
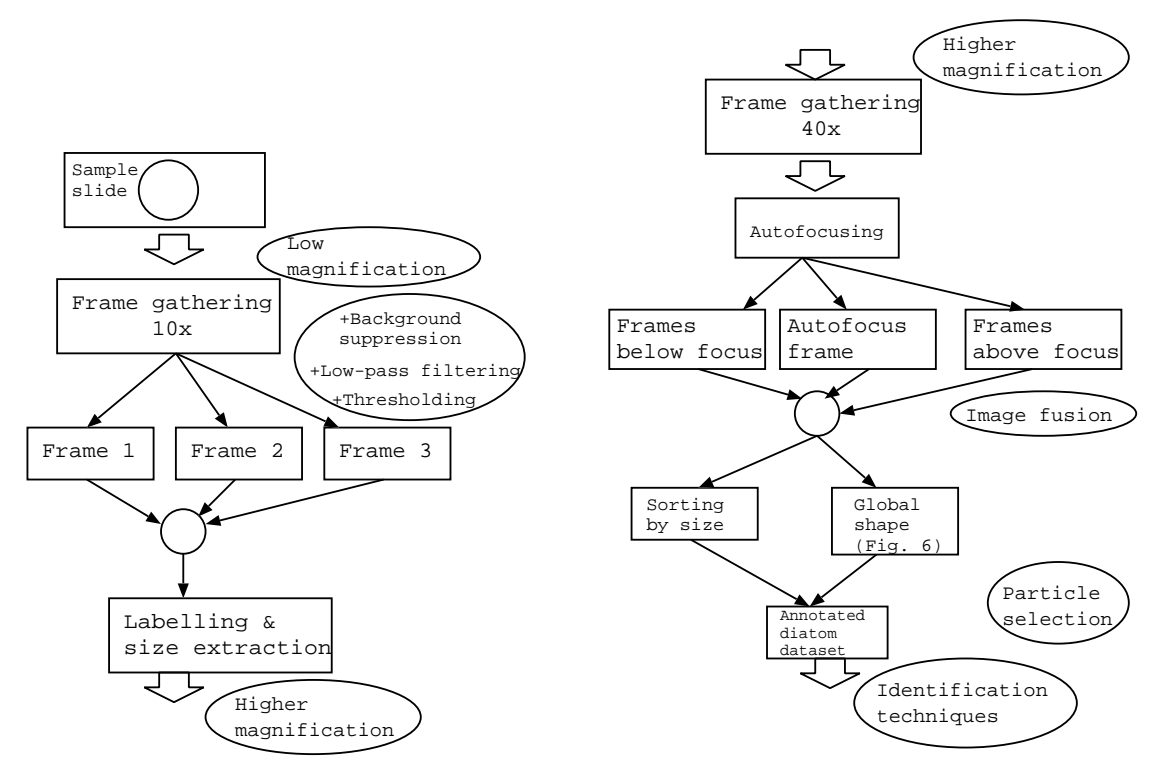

Figure 2. Schematic overview of the automatic slide scanning. Left: processing stages at low magnification. Right: processing stages at higher magnification. Note: the screening of particles using phase correlation techniques is performed at medium magnification (i.e. 20x). Screening using shape descriptors is applied at higher magnification.

\subsection{Calibration}

A first operation, only required once before the automatic scanning process, is the calibration of the diatom size at different magnifications. Figure 4 shows images of a microscopic ruler taken with different lenses. The ruler has a length of $1 \mathrm{~mm}$ and it is divided in 100 divisions of $10 \mu \mathrm{m}$ each. Hence, it is possible to determine the number of pixels between the ruler's divisions at all magnifications. The calibration allows us to obtain real sizes by converting pixels to microns. 

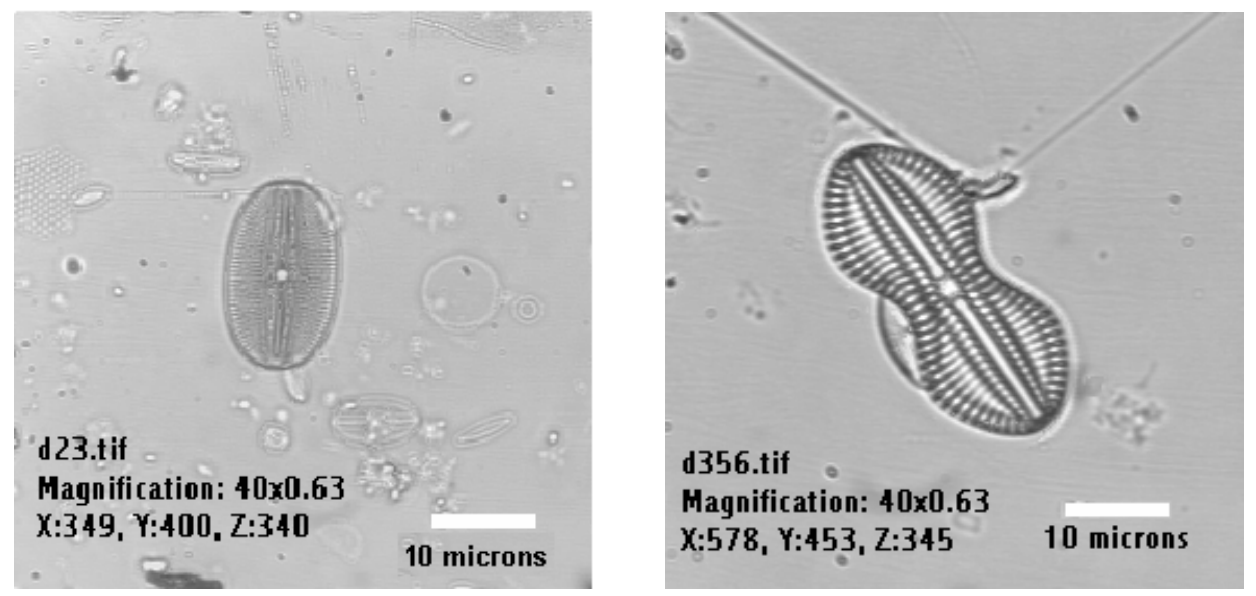

Figure 3. Annotated particles provided by the automatic system. Each particle detected at low magnification is later visualized at higher magnification and presented at the center of the image. Each particle is annotated with its spatial position, depth and magnification.

\subsection{Slide scanning at low magnification}

At low magnification it is necessary to arrange all the fields as a multi-frame mosaic that allows us to identify the positions of "candidate particles." With the equipment described in Section 2, an entire slide results in an array of frames, in which each frame covers an area of $500 \times 500 \mu \mathrm{m}$. Figure 5 shows a subset of a mosaic at low resolution. Such a mosaic provides a panoramic view with the relative and absolute particle coordinates necessary for further analysis.

\subsection{Algorithms for particle detection}

The detection is based on the use of histogram thresholding, such that we obtain the centroid position and the size of all particles. We should point out that it is difficult to discriminate between diatoms and non-diatom objects at this resolution (Fig. 5). The main goal is to get an estimate of the centroid position of the particle that will be further analyzed at higher magnification. Below, we give a detailed description of the algorithms used (schematically shown in Fig. 2). The operations listed below are illustrated by a series of images shown in this section.

- Background correction: In general it is almost impossible to obtain homogeneous illumination of all frames. This effect is caused by the optics of the microscope, but can be eliminated by a background suppression method using a "rolling ball" algorithm (Sternberg, 1983; Russ, 1995). Figure 6 (top) shows one frame from a mosaic after binarization, but without background correction. We can observe that the effect of inhomogeneous illumination is more pronounced. The binarization on the basis of selecting a threshold value $\mathrm{T}$ located midway between the histogram extrema (see inset in the lower-left corner) is not efficient. To solve this problem, we perform two pre-processing operations on the original image. The first one is 


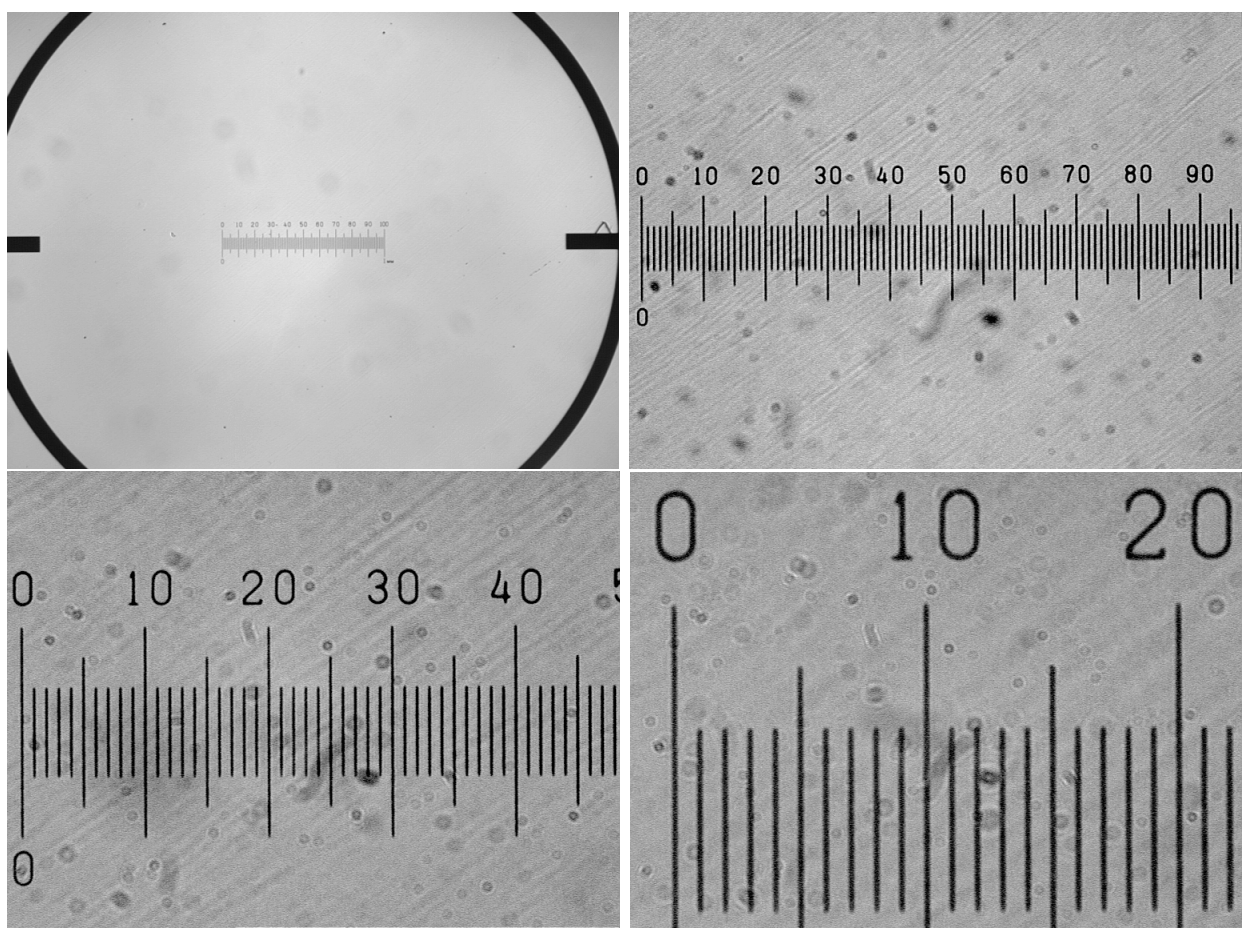

Figure 4. Images of a microscopic ruler at different magnifications. Total size of the ruler is $1 \mathrm{~mm}$, with 100 divisions of $10 \mu \mathrm{m}$. Top-left: $2.5 \mathrm{x}$ lens; top-right: $10 \mathrm{x}$; bottom-left: $20 \mathrm{x}$ and bottom-right: $40 \mathrm{x}$. Ocular magnification: $0.63 \mathrm{x}$.

a background correction with the "rolling ball" technique. The idea behind this algorithm is very simple. Consider the image as a 3D surface, where the height is given by the pixel values. Then imagine a ball underneath the surface, and roll the ball under the entire image. The position of the top of the ball corresponds to the background that can be subtracted from the image. An important parameter is the size of the ball. The results shown here were obtained by using a mask of $25 \times 25$ pixels containing an hemisphere. An alternative solution is to use a flat fielding technique by dividing (or substracting) the original image with a lowpass filtered version of itself, using a filter with a very large window size. See for example Seul et al. (2000).

- Lowpass filtering: Because we must discriminate between the foreground (particles) and background, a thresholding is required. However, the histogram of the image after background correction shows multiple peaks that complicate the selection of a threshold value. A simple smoothing operation, by neighborhood averaging, results in a histogram with two distinct peaks, and this facilitates the detection of the valley that segregates foreground from background. The lowpass filter used is a straightforward $3 \times 3$ convolution mask, with the nine elements equal to $1 / 9$. We note that the resulting contours of the particles do not necessarily correspond 


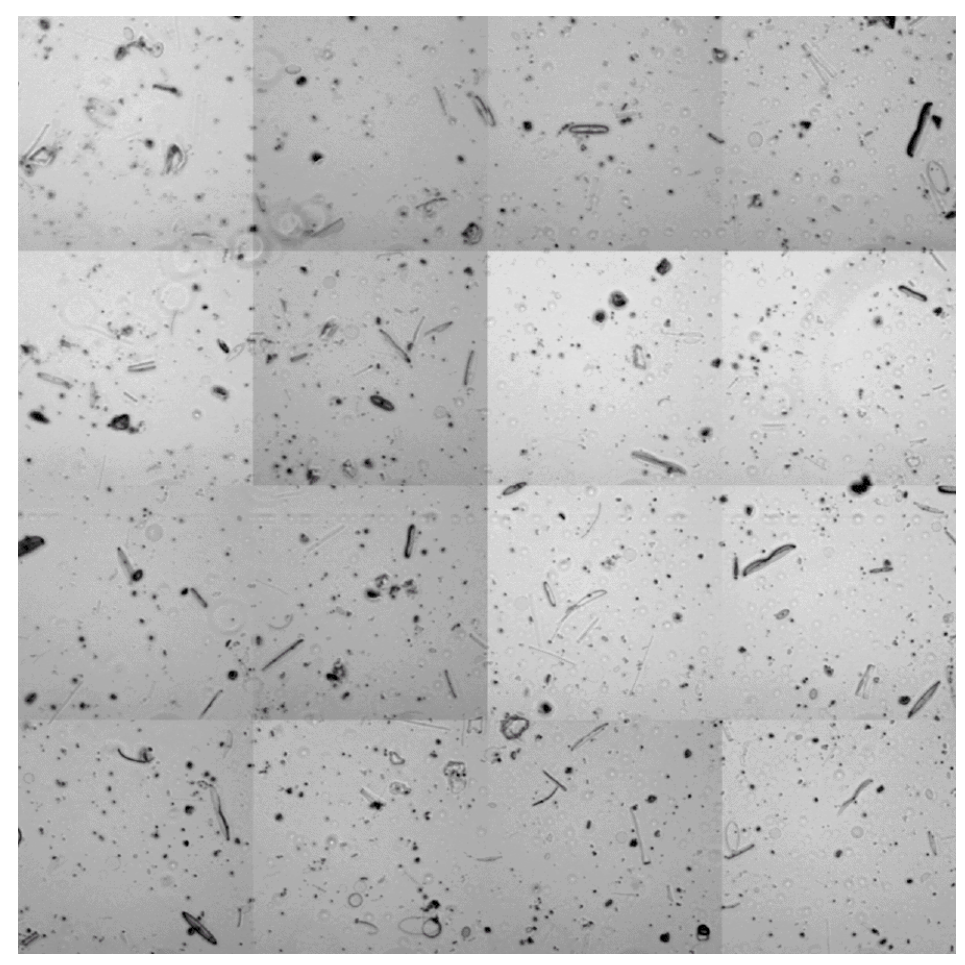

Figure 5. Example of a mosaic of images at low resolution (magnification 10x). The size of each frame is $500 \times 500 \mu \mathrm{m}$; the total area is $2 \times 2 \mathrm{~mm}$. The effect of inhomogeneous illumination between frames can be observed; this can be eliminated using a background suppression algorithm.

well to the particles, which is due to the smoothing. However, this not a problem because only the particles' centroid position is required for a further analysis at higher magnification.

- Binarization: A few methods have been proposed for binarization by thresholding; for a good summary see Kindratenko (1997). The most important is to find a good threshold value, but there is no solution that suits all applications, i.e. the best method needs to be found empirically. We found that the triangle algorithm (Zack et al., 1977) provides a simple and efficient solution. The threshold value is determined from the histogram by computing the intersection of the perpendicular line to the line that joins the global maximum of the histogram with the lowest gray level (see Fig. 7). This technique is particularly effective in the case of objects that produce weak peaks in the histogram (Biemond and Merserau, 1998). Figure 8 (top) shows the result after applying the triangle algorithm.

- Combining images: In order to improve the detection of particles that are located at different focal planes, we do not only capture the best focused low magnification image, but also a small number of images above and below the best-focused one. These can be combined so that most particles in a slide can be detected in one pass. To this end we average the best-focused image with the two neighboring ones, 


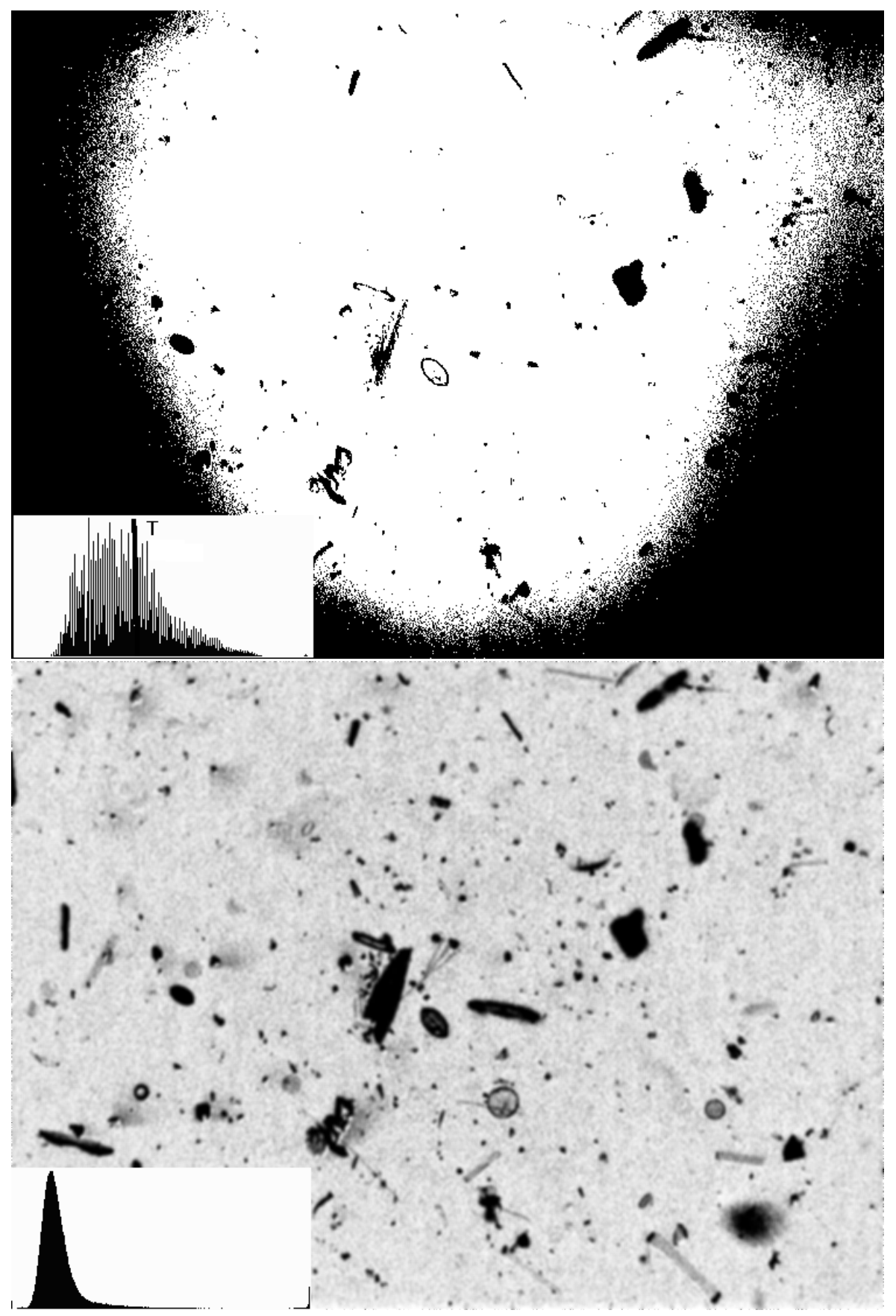

Figure 6. Top: histogram thresholding. Note the inhomogeneous illumination effect. Bottom: result of the background correction (by rolling ball) and lowpass filtering. Note the change of the histogram shown in the lower-left corner. 


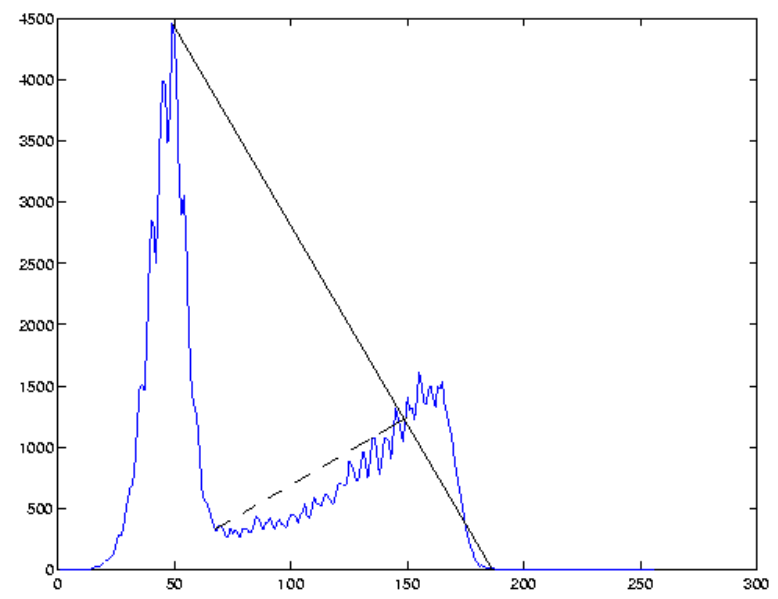

Figure 7. Triangle thresholding algorithm. A line is constructed between the maximum peak of the histogram and the highest gray level. The maximum distance from the histogram to this line (dashed) provides the threshold value, in this case 67.

separated by a distance of $10 \mu \mathrm{m}$. Figure 8 (bottom) shows the final result of the binarization process, after the processing described above. This procedure allows for efficient particle detection, even in the case of particles that have extreme gray levels (see the rod-shaped particle at bottom-right side of Fig. 8 (bottom), labeled with \#92). The method for improving the detection by combining images from different depths is illustrated in Figs 9 and 10. Figure 9 shows the binarized low magnification images below and at the best focus plane, respectively. Figure 10 shows the images above the best focus plane as well as the fused image after averaging the three multifocus images. Hence, this very simple technique allows the detection of particles that otherwise might have been skipped.

\subsection{Techniques for particle screening}

Even at medium magnification it is difficult to distinguish between diatoms and debris (see Fig. 11). The smallest particles cannot be skipped, because they might correspond to tiny diatoms that cannot be resolved with the lenses described in Section 2. Even a diatom expert cannot make a decision by looking at such images. Therefore, we are considering three main strategies for removing candidate particles, and therefore debris rejection.

- Image gathering guided by size: We implemented particle localization guided by size, i.e. a selective search process for detecting particles that fall within a specific size range. This simple method allows us to create different image catalogues indexed by size, but it does not allow us to eliminate debris. It should be combined with other methods based on shape or symmetry, for example, in order to improve the discrimination. Figure 12 shows an example of indexing by size. 


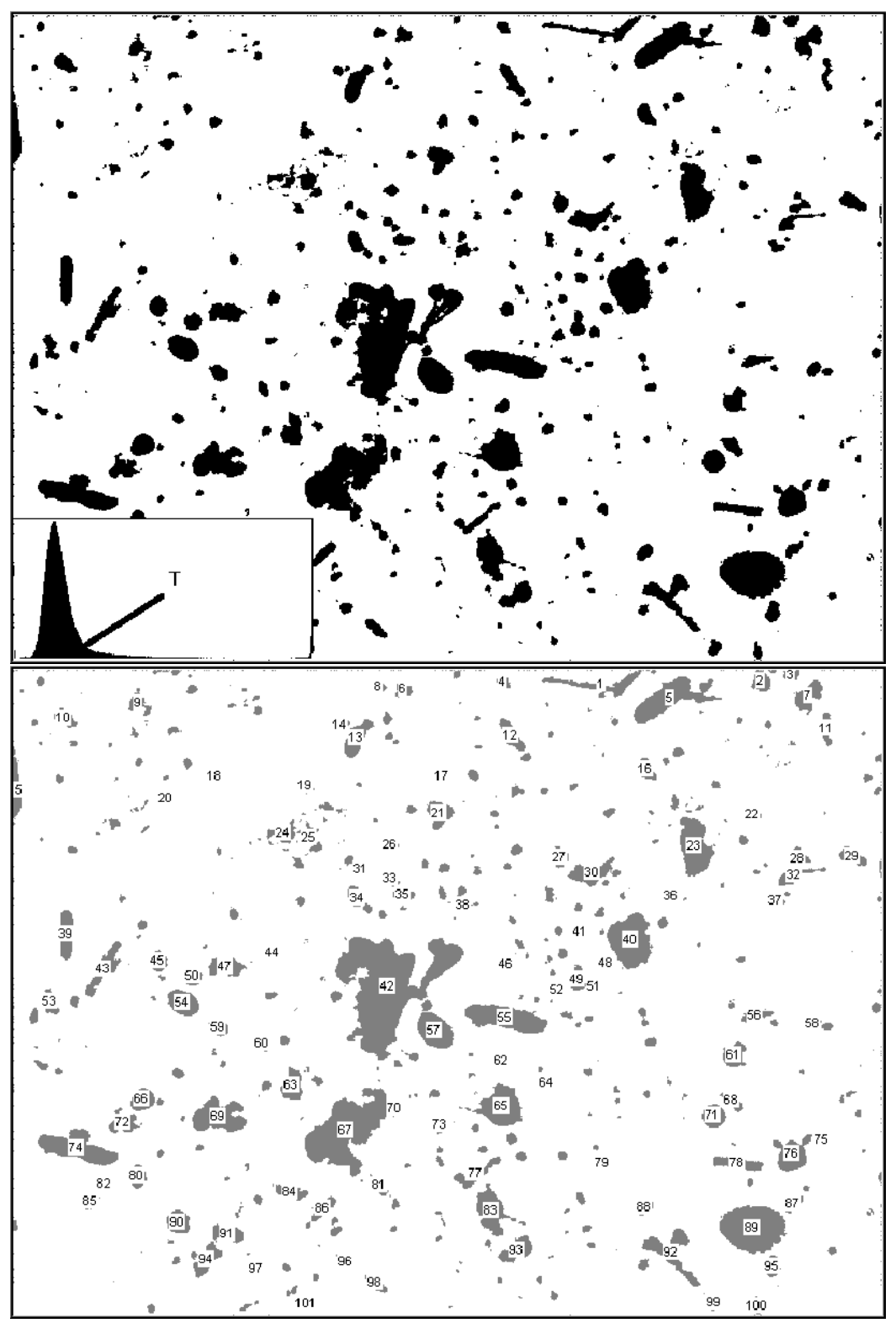

Figure 8. Top: binarized image using the "triangle" thresholding. Note the adaptive threshold value marked by an arrow line (lower-left corner). Bottom: labeled particles.

- Image gathering using shape descriptors: The main difficulty for automating slide scanning is that at low (or even medium) magnification it is difficult to distinguish candidate diatoms from debris. One possible solution is to apply an analysis at high magnification, e.g. using a combination of shape and symmetry descriptors, 


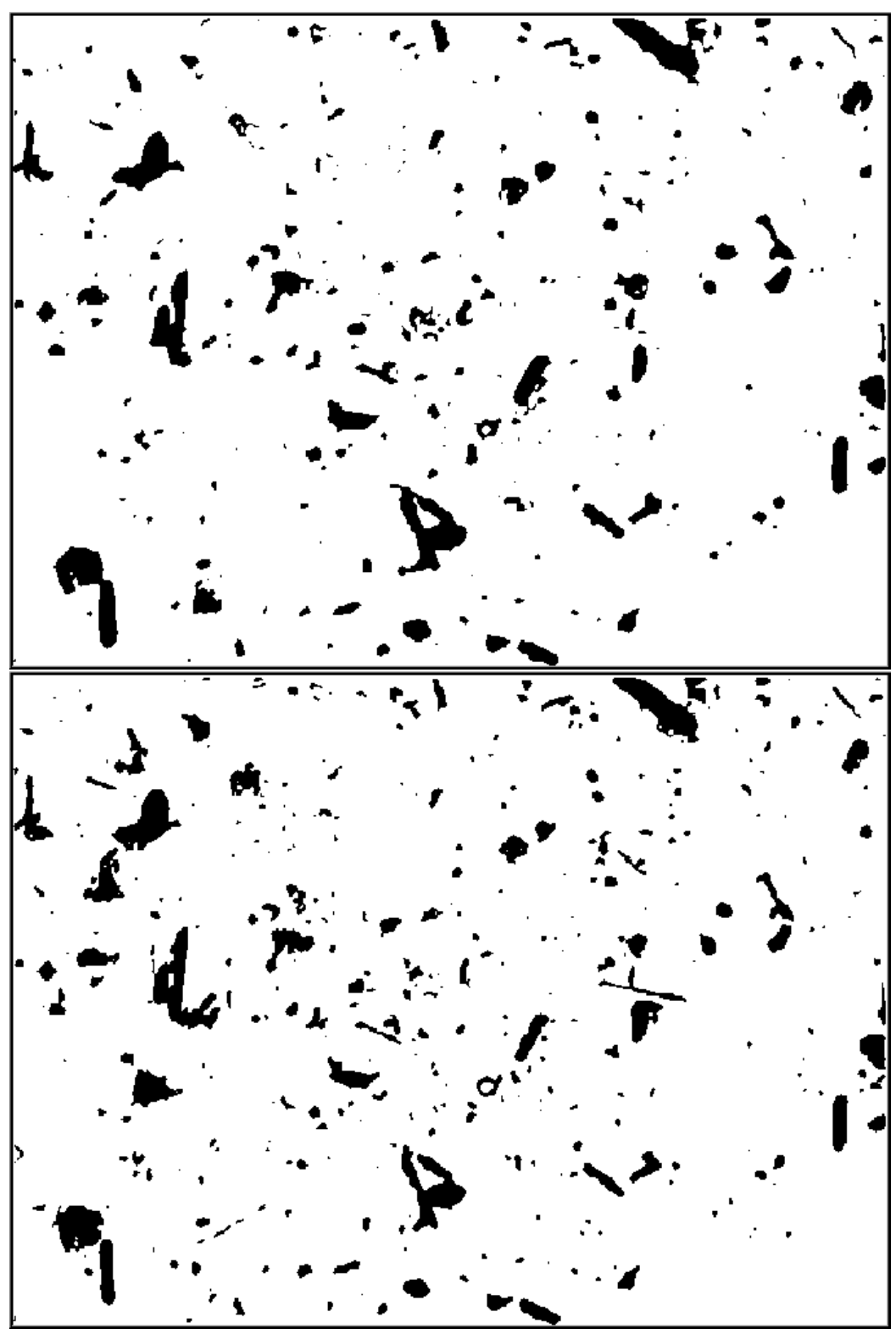

Figure 9. Top: binarized, low magnification image from below the best focused plane; bottom: binarized autofocus plane.

and only acquiring the particles that pass the tests. This approach provides a more effective and flexible screening technique, because we can establish a multicriteria decision-rule system based on both local and global shape descriptors. There are some methods based on symmetry detection (e.g. based on the gradient of the 


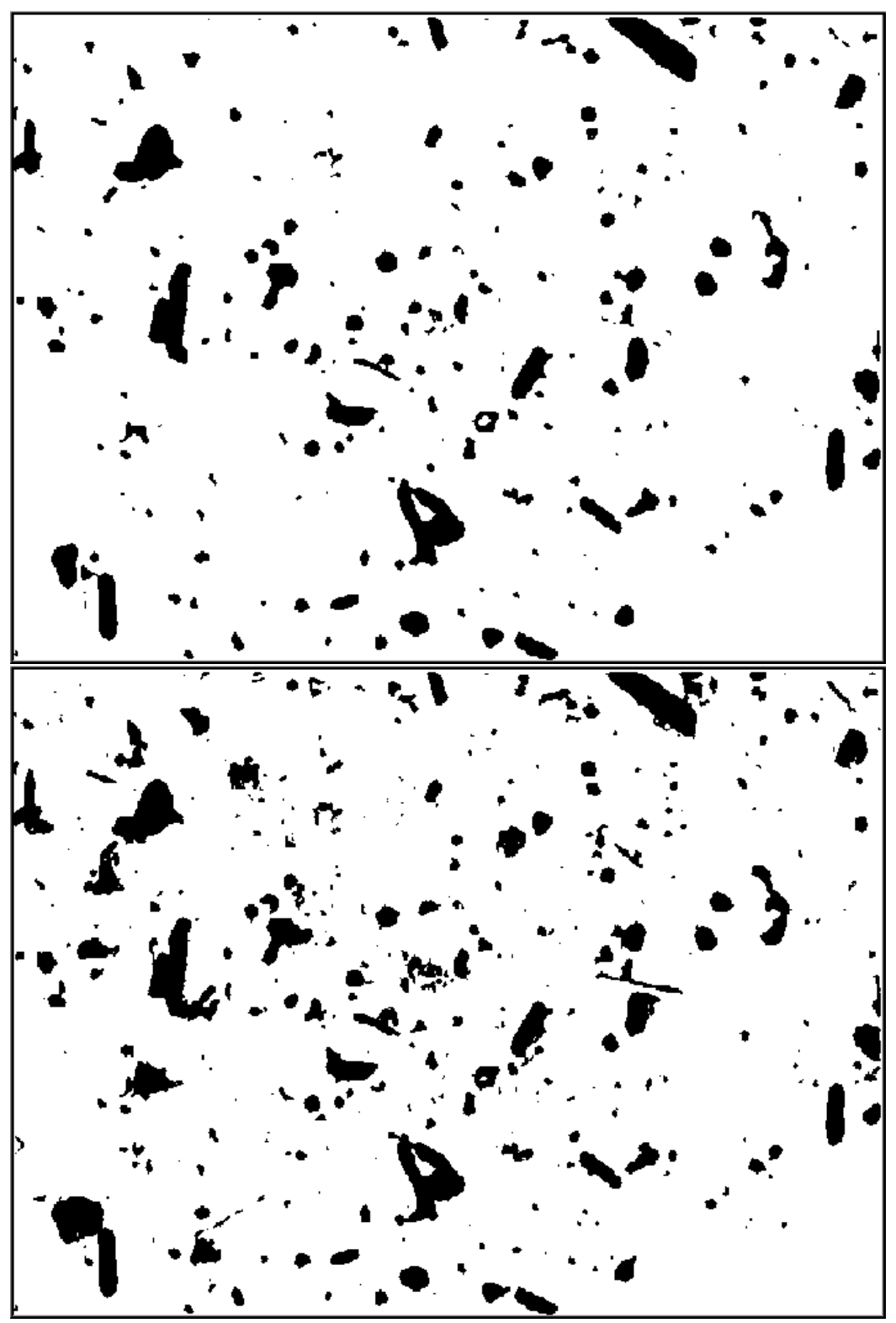

Figure 10. Top: binarized, low-magnification image from above the best focus plane; bottom: the fused image obtained by averaging the three images shown in Fig. 9 and here (top).

histogram, see Sun, 1995), geometric descriptors (circularity, elongation, etc., see Jain and Hong, 1996), and global shape descriptors (e.g. Hough transform). We have tested an algorithm for the detection of elliptic shapes using the Randomized Hough Transform as described by McLaughlin (2000). The main idea here is to 


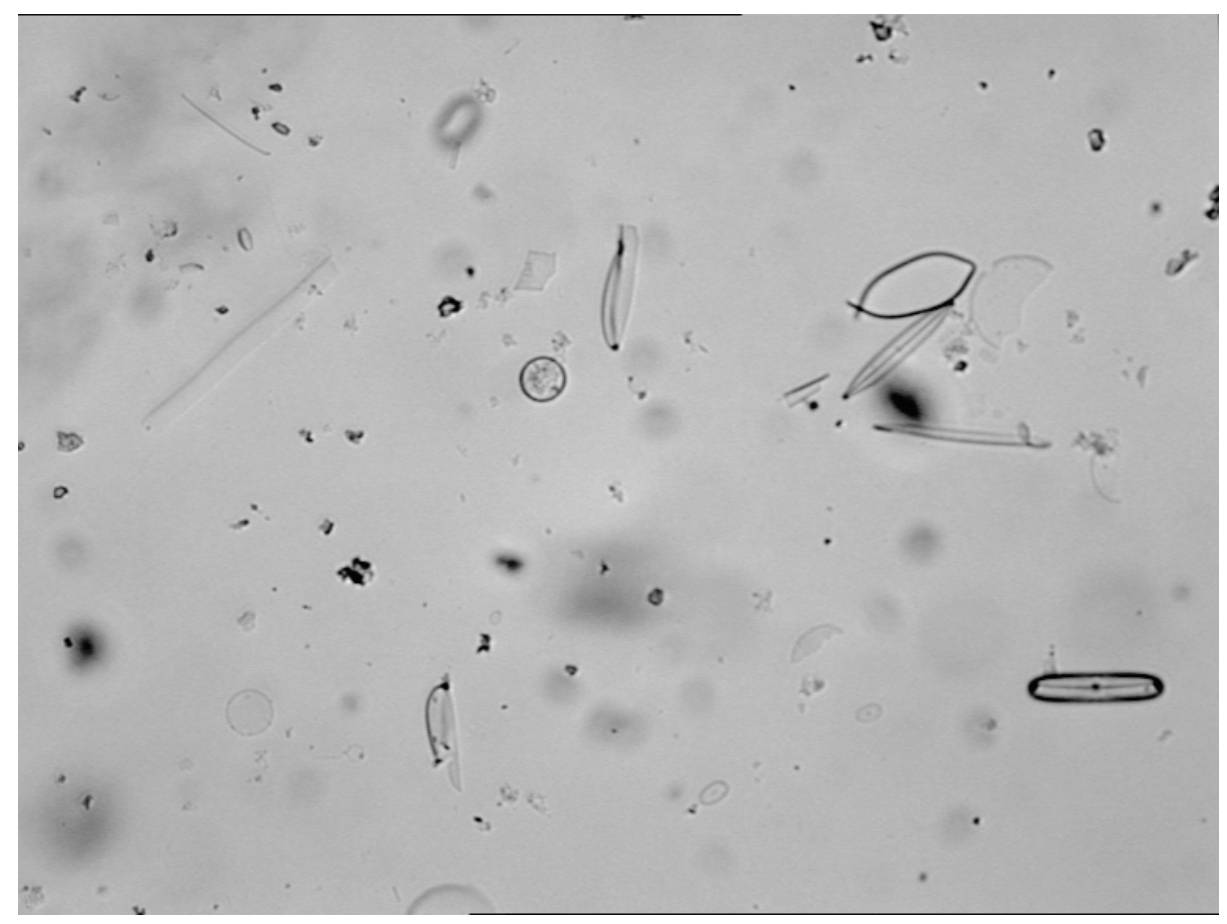

Figure 11. Example of a microscopic image at medium magnification (20x). Note the difficulty in discriminating between diatoms and debris. Image size: $768 \times 576$ pixels.

apply an edge detector, such as the Sobel gradient, which will be described in Section 4, and to detect (pseudo-)elliptical shapes via the Hough transform. Figure 13 shows examples. Purely elliptical pennate diatoms can be detected and circular (centric) ones as well. One problem of the Hough transform is that it costs a lot of CPU time. The percentage of usable valves that can be detected with this method remains to be estimated. There are many diatoms with shapes that do not resemble simple ellipses. In such cases the screening process may require some of the analysis methods described in the identification chapters in this book. A very recent book about shape analysis has been written by da Fontoura and Marcondes (2001).

- Image screening using phase-only correlation filtering: this is the only technique that can be directly applied to medium magnification images (i.e. $20 \mathrm{x}$ ), see Fig. 11. Each particle detected at this magnification is padded with a uniform gray level to obtain an image of $256 \times 256$ pixels (Fig. 14). The screening is accomplished by matching a prototype image and the test image with the particle. Image matching is defined as the problem of evaluating the similarity of objects in different images (Chen and Defrise, 1994). This technique has been used in many applications. Here, it will allow us to verify the presence of a diatom in a captured image. Let $T(x, y)$ be the template image and $S(x, y)$ a sample image to be analyzed. The technique that we are using here is referred to as symmetric phaseonly matched filtering (SPOMF) in the literature. It is based on computing the 


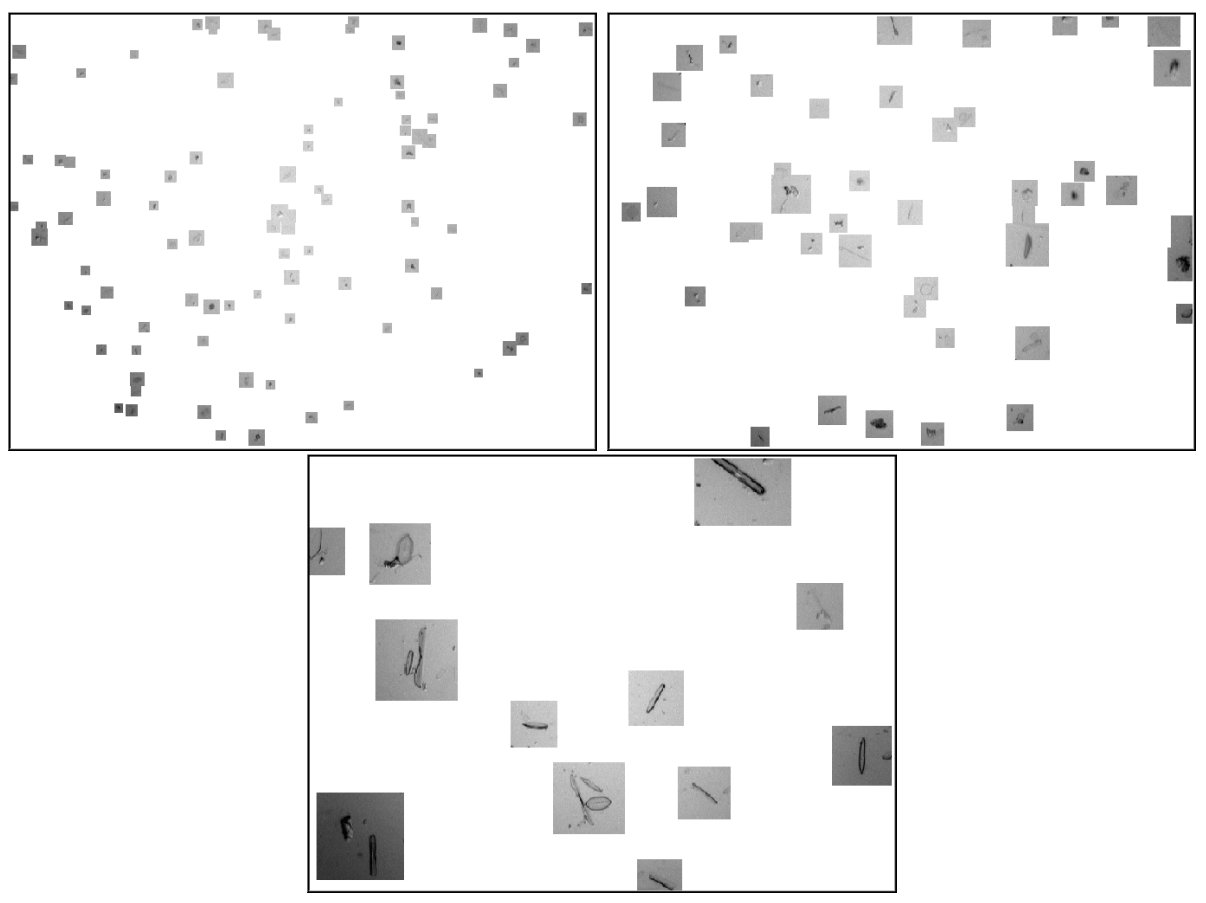

Figure 12. Image indexing by size. Size ranges between $10-20 \mu \mathrm{m}$ (top-left), $20-50 \mu \mathrm{m}$ (top-right) and $50-120 \mu \mathrm{m}$ (bottom).

phase spectra of the two images and then the phase-only matched filtering (e.g. de Castro and Morandi, 1987). However, the spectral phase is not scale and rotation invariant. Matching of scaled and rotated images can be done by substituting the Fourier transform with the Fourier-Mellin transform, as has been shown by Chen and Defrise (1994), or with the scale transform as proposed by Pech-Pacheco et al. (2000). The phase-only matched filtering algorithm using the Fourier transform is as follows:

\section{Algorithm 1: Phase-only correlation filtering}

1. Compute the Fourier transform $\mathcal{T}(u, v)$

2. Extract the spectral phase $\mathcal{P}(\mathcal{T}(u, v))=\exp \left[\mathrm{j} \phi_{T}(u, v)\right]$

3. Compute the Fourier transform $\mathcal{S}(u, v)$

4. Extract the spectral phase $\mathcal{P}\left(\mathcal{S}^{*}(u, v)\right)=\exp \left[-\mathrm{j} \phi_{S}(u, v)\right]$

5. Compute the inverse Fourier transform of the phase product:

$$
C(x, y)=\mathcal{F}^{-1}\left[\frac{\mathcal{T}(u, v)}{|\mathcal{T}(u, v)|} \frac{S^{*}(u, v)}{\left|S^{*}(u, v)\right|}\right]=\mathcal{F}^{-1}\left[\exp \left\{\mathrm{j}\left[\phi_{\mathcal{T}}(u, v)-\phi_{\mathcal{S}}^{*}(u, v)\right]\right\}\right]
$$




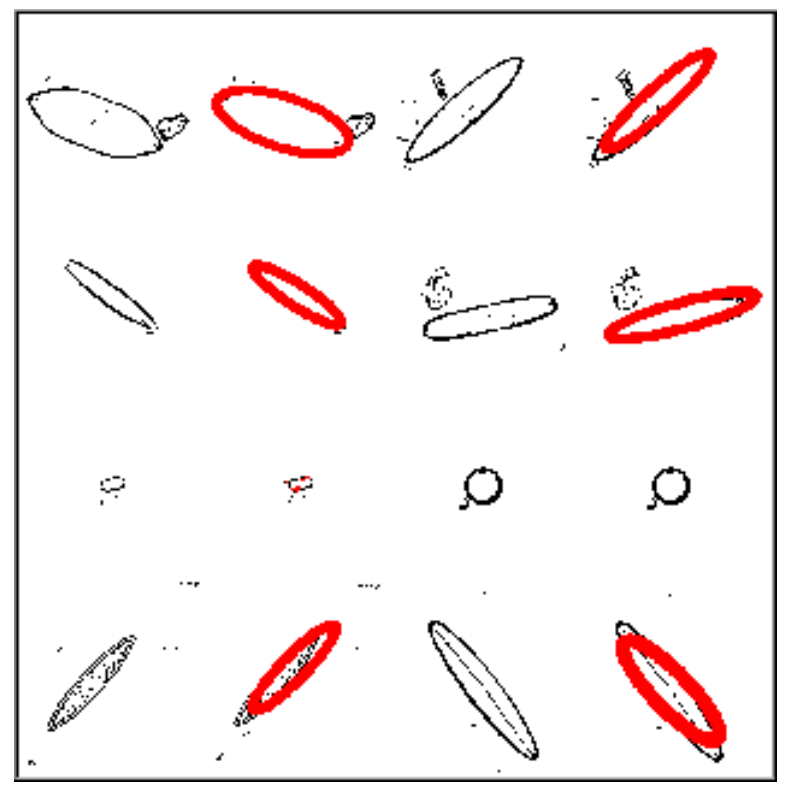

Figure 13. Ellipse detection through the Hough transform. The input for the Hough transform (first and third columns) are edges detected by the Sobel masks (see Section 4). The second and fourth column show the results of the ellipse detection. The third particles in the first and third column are debris and the method does not detect any feature.

6. Threshold the magnitude of $C(x, y)$ to detect the maximum peaks (eliminating false alarms).

Here $\phi_{\mathcal{S}}^{*}$ represents the complex conjugate of the phase $\phi_{\mathcal{S}}$.

As a result, the SPOMF method produces a sharp correlation peak at the position where the images match. Figure 14 shows some of the particles detected by the method of indexing by size, as described in Section 3.5. Figure 15 shows the results of the SPOMF correlation method using as a template the top-left image in Fig. 14. A significantly higher correlation peak can be observed in the case of a diatom (Fig. 15, middle) if compared with the case of debris (Fig. 15, bottom). A first estimate is that we can detect about $80 \%$ of usable valves. This is a very significant rate in view of the magnification of $40 \mathrm{x}$. The same process can be applied at higher magnifications, but would cost more CPU time.

\section{Autofocusing}

Several autofocusing methods have been proposed in the literature (Groen et al., 1985; Krotkov, 1987; Firestone et al., 1991; Subbarao et al., 1993; Yeo et al., 1993; Bocker et al., 1996). These methods use different focus functions, which measure the relative sharpness of images taken at different depths. The depth at which the function has the largest value will give the best-focused image. In the next sections we will review different focus functions. Let $I(n, m)$ be an image of size $N \times M$ 


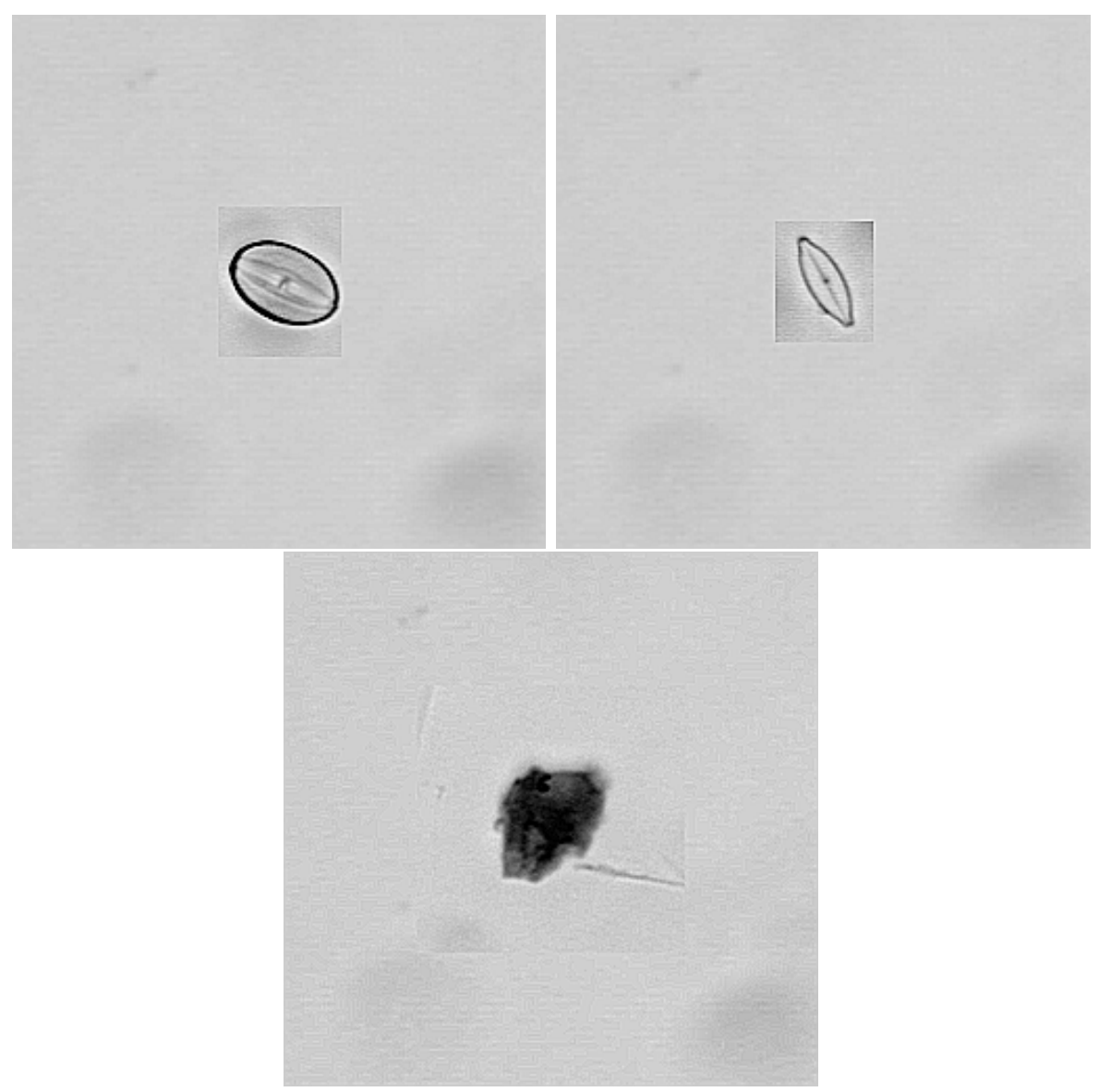

Figure 14. Top-left: diatom image used as template for the phase-correlation process. Top-right and bottom: two particles detected by the method of indexing by size as described in Section 3.5.

and let us assume a stack of $k$ images taken by changing the focal depth in steps of $1 \mu \mathrm{m}$. Figure 16 shows images from a stack above and below the best-focused image shown at the center.

\subsection{Focus function based on image contrast}

A well-focused image can be expected to show strong variation in gray levels. The local variance at point $(n, m)$, with $n=1 \ldots N$ and $m=1 \ldots M$, is given by

$$
F_{l v}(n, m)=\frac{1}{w_{x} w_{y}} \sum_{i}^{w_{x}} \sum_{j}^{w_{y}}[I(n+i, m+j)-\bar{I}]^{2},
$$



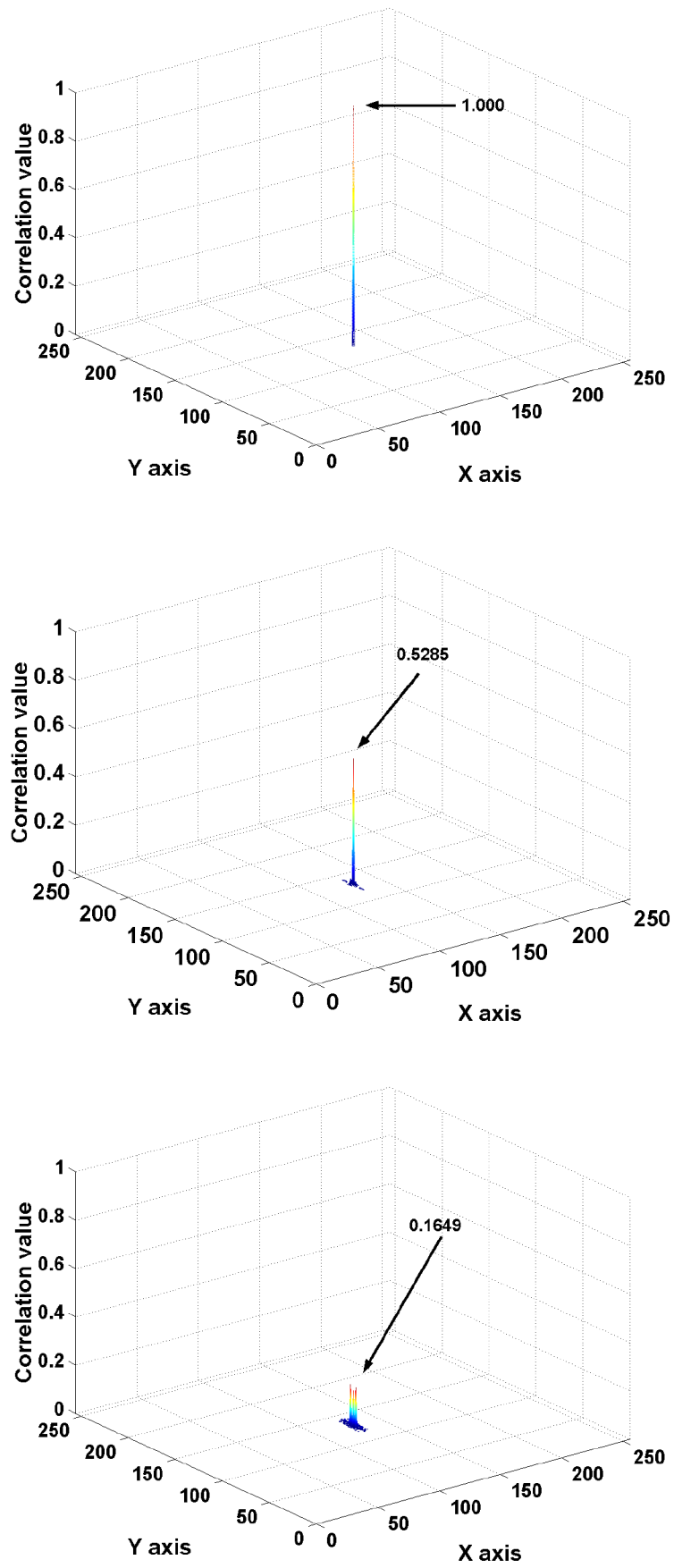

Figure 15. Top: the phase-only autocorrelation peak of the diatom template (Fig. 14, top-left) has a value of 1.0. Middle: correlation peak of the template (Fig. 14, top-left) with the diatom shown in Fig. 14 (top-right). Bottom: correlation peak corresponding to the particle shown in Fig. 14 (bottom). 
where $\bar{I}$ is the mean gray level value

$$
\bar{I}=\frac{1}{w_{x} w_{y}} \sum_{i}^{w_{x}} \sum_{j}^{w_{y}} I(n+i, m+j)
$$

and $w_{x} \times w_{y}$ is the size of a window centered on the point $(n, m)$. A global variance can be defined from the local variance by

$$
F_{v a r}(I)=\frac{1}{N M} \sum_{n}^{N} \sum_{m}^{M}\left[F_{l v}(n, m)-\bar{F}_{l v}\right]^{2},
$$

where $\bar{F}_{l v}$ is given by

$$
\bar{F}_{l v}=\frac{1}{N M} \sum_{n}^{N} \sum_{m}^{M} l v(n, m) .
$$

\subsection{Focus functions based on differentiation}

\subsubsection{First derivative methods}

A well-focused image is also expected to have sharper edges, hence image gradients are applied for computing focus measures. Given a gradient image, the focus measure has to pool the data at each point. Tenenbaum (1970) proposed a method, called Tenengrad, that is considered a benchmark in this field (Schlag et al., 1983; Krotkov, 1987). This method estimates the gradient magnitude at each image position and sums all magnitudes exceeding a threshold $T$. The Sobel operator uses the two convolution masks

$$
S_{x}=\left(\begin{array}{rrr}
-1 & 0 & 1 \\
-2 & 0 & 2 \\
-1 & 0 & 1
\end{array}\right) \quad S_{y}=\left(\begin{array}{rrr}
1 & 2 & 1 \\
0 & 0 & 0 \\
-1 & -2 & -1
\end{array}\right)
$$

and the gradient's magnitude is calculated as

$$
S(n, m)=\sqrt{\left[G_{x}(n, m)\right]^{2}+\left[G_{y}(n, m)\right]^{2}},
$$

where $G_{x}(n, m)$ and $G_{y}(n, m)$ are the convolutions of the input image $I(n, m)$ with the masks $S_{x}$ and $S_{y}$. The Tenengrad focus measure is given by

$$
F_{t e n}(I)=\sum_{n}^{N} \sum_{m}^{M}[S(n, m)]^{2} \quad \text { for } \quad S(n, m)>T .
$$

\subsubsection{Boddeke's algorithm}

Boddeke et al. (1994) and Boddeke (1998) proposed also a very simple focus measure. This method is based on applying a $(-1,0,1)$ filter mask only along the horizontal $(n)$ axis of an image stack represented by $I(n, m, z)$. The focus function is defined by squaring and adding all the filtered pixel values, i.e.

$$
F(z)=\sum_{n, m}[I(n+1, m, z)-I(n-1, m, z)]^{2} .
$$


The method is extremely simple and provides a single, sharp peak (Ellenberger and Young, 1998).

\subsubsection{Second derivative methods}

The use of a second derivative operator is another technique for analyzing the high spatial frequencies associated with sharp edges. As a second derivative operator we use the Laplacian, which can be approximated using the filter mask

$$
L=\frac{1}{6}\left(\begin{array}{rrr}
0 & -1 & 0 \\
-1 & 4 & -1 \\
0 & -1 & 0
\end{array}\right)
$$

We use two methods for pooling the data at all positions. The first one is the sum of all the absolute values, giving the following focus measure:

$$
F_{l a p}(I)=\sum_{n}^{N} \sum_{m}^{M}|L(n, m)|
$$

where $L(n, m)$ is the convolution of the input image $I(n, m)$ with the mask $L$. The second method consists of computing the variance, see below.

\subsection{New focus functions based on differentiation}

\subsubsection{Variance of gradient}

The alternative for pooling the gradient information is to calculate the variance of gradient magnitudes, i.e. the variance of the magnitude of the Sobel gradient. The reason for this approach is to define a more discriminative measure, similar to a second derivative (Laplacian), but increasing the robustness to noise (Pech-Pacheco et al., 2000; Pech-Pacheco and Cristobal, 2001). Figure 16 shows a comparison of focus measurements without and with noise. The new focus measure is given by

$$
F_{\text {sob_var }}(I)=\sum_{n}^{N} \sum_{M}^{m}[S(n, m)-\bar{S}]^{2} \quad \text { for } \quad S(n, m)>T,
$$

where $\bar{S}$ is the mean of magnitudes given by

$$
\bar{S}=\frac{1}{N M} \sum_{n}^{N} \sum_{m}^{M} S(n, m) .
$$

\subsubsection{Variance of Laplacian}

In the second new method we calculate the variance of the absolute value of the Laplacian, i.e.

$$
F_{\text {lap_var }}(I)=\sum_{n}^{N} \sum_{m}^{M}(|L(n, m)|-\bar{L})^{2}
$$


where $\bar{L}$ is the mean of absolute values given by

$$
\bar{L}=\frac{1}{N M} \sum_{n}^{N} \sum_{m}^{M}|L(n, m)| .
$$

\subsubsection{Window size selection}

An important parameter is the window size for computing the variance and gradient. We have experimented with 23 different stacks of multifocus images taken at high magnification (40x). Each stack is composed of 100-150 images. Figure 17 (top) shows the focus estimate of all the stacks tested as a function of the window size. Level zero in the plot indicates the optimum focus plane. The variance-only method provides a better focus estimate for small windows, but it costs more CPU time for large windows. The vertical bars are shorter for small windows, meaning a better focus estimate. However, the variance-only method lacks robustness, because, as can be seen from the dash-dotted plot in Fig. 16, there are situations in which it yields a wrong focus estimate. Figure 17 (bottom) shows that the variance of Sobel provides better results for large windows, which is an advantage because of its smaller CPU time. The vertical bars are shorter for large window sizes, hence it provides a better focus estimate. Therefore, we decided to use the variance of Sobel for the full image size (global variance), because it has the lowest computational cost.

\subsection{Assessment of focus measures}

Here we will assess the shape of the focus measures shown in Fig. 16. Due to the lack of a quantitative, standard metric for assessing the shape of the focus curves, we propose two new measures based on sharpness and smoothness.

- Sharpness: The sharpness of the peak is not the only criterion for selecting a good focus measure. In fact, as Subbarao et al. (1993) have pointed out, any focus measure can be artificially sharpened, simply by squaring the focus measure. Kurtosis is a candidate for "peakedness." However, it suffers from a high noise sensitivity. We are more interested in having a sharp autofocus curve, in order to reduce the number of images to fuse by means of multifocus fusion techniques described in the next section. We define a sharpness metric as the average value of the width $d$ of the focus curves at three equidistant focus levels from the maximum value, i.e. the width of the focus curves at values of $0.25,0.5$ and 0.75 (see Fig. 16):

$$
F_{1}=k_{1} \sum_{i=1}^{3} d_{i},
$$

where $d_{i}$ is the width at the three levels mentioned above and $k_{1}$ is a normalizing constant. Table 1 shows sharpness measures (normalized with respect to the Sobel+variance value). It can be seen that the Sobel+variance and Tenengrad methods provide the smallest $F_{1}$ values.

- Smoothness and noise sensitivity: We define a relative measure based on the accumulative sum of the absolute gradient value of the focus measure (Fig. 16) with 

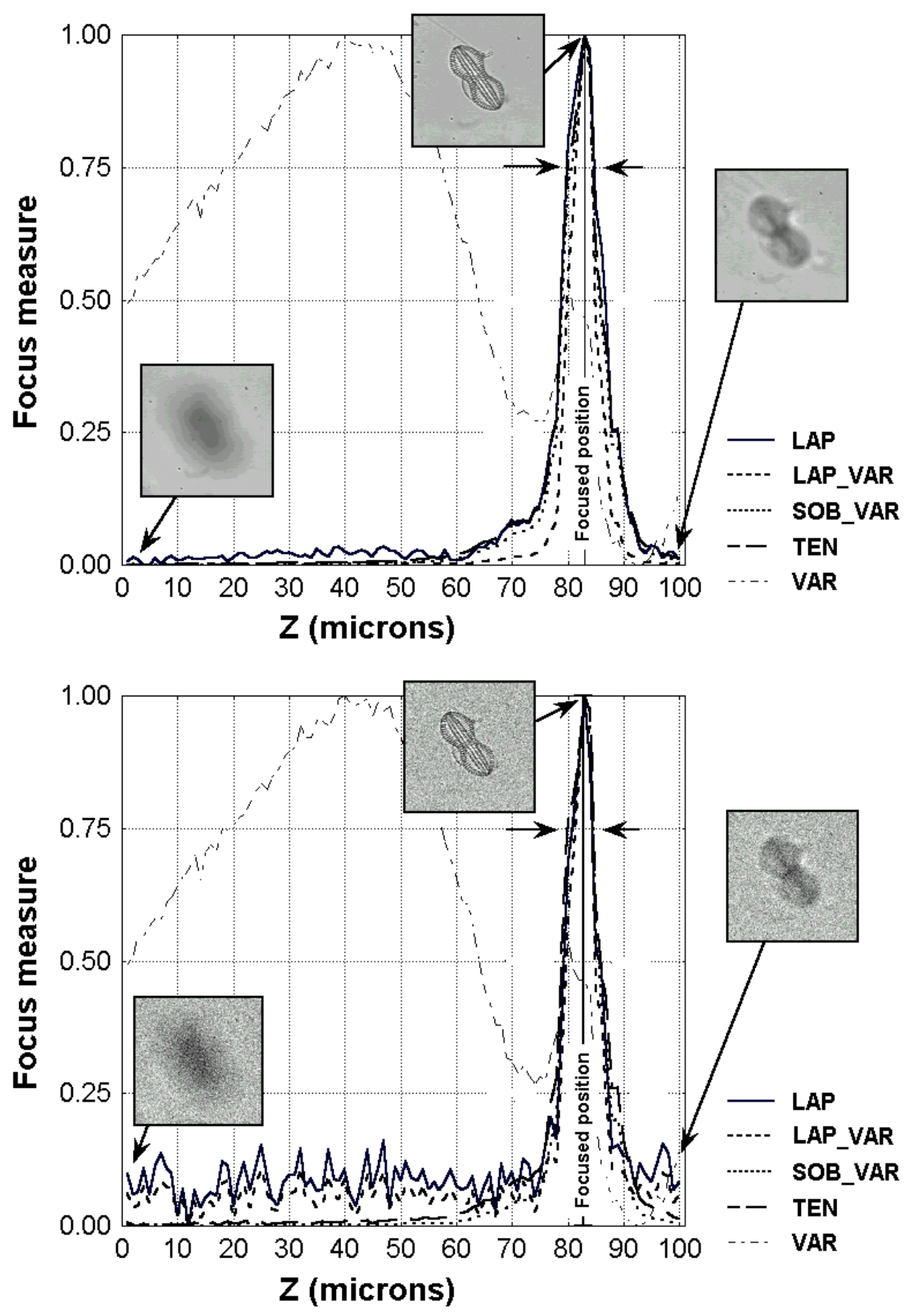

Figure 16. Top: comparison of focus measurements; no noise. Bottom: the same for noisy data. LAP: Laplacian; LAP_VAR: variance of Laplacian; SOB_VAR: variance of Sobel; TEN: Tenengrad; VAR: global variance. Note that the global variance method (VAR) provides a wrong estimate of the best-focused image for this image stack. The images located above the horizontal arrows are the ones to be selected for multifocus fusion; see Fig. 18. 

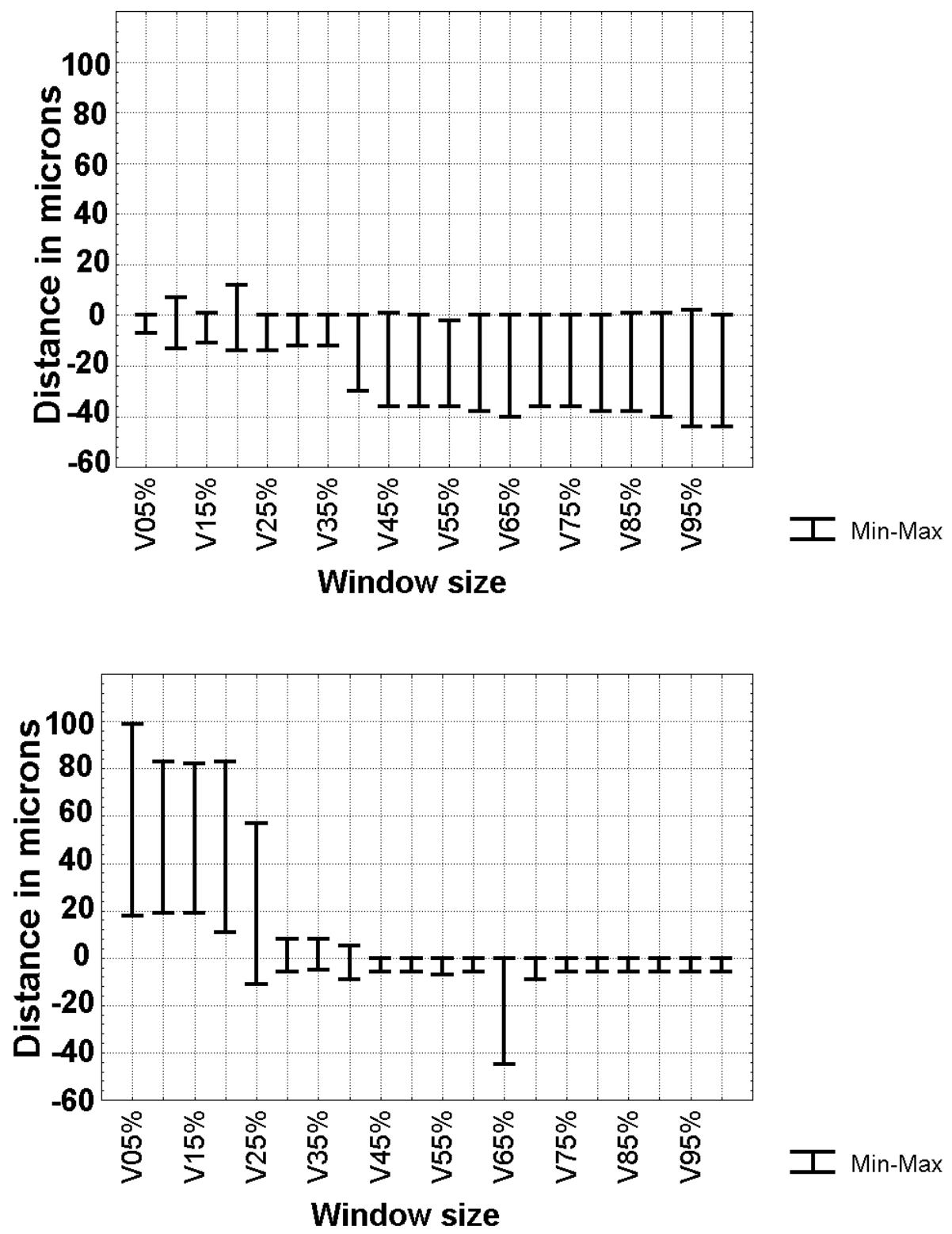

Figure 17. Window size evaluation from 23 different stacks of multifocus images. Each stack consists of 100-150 multifocus images. The horizontal axes of the plots represent the size percentage of the window used for the computation in relation to total image size. Level 0 at the vertical axes means the best focusing plane. Top: variance measure; bottom: variance of Sobel measure. 
Table 1. Sharpness and smoothness results.

\begin{tabular}{lcccc}
\hline \hline function & Sobel+var & Tenengrad & Laplacian & Lapl+var \\
\hline$F_{1}$ & 1 & 1.21 & 3.79 & 3.28 \\
$F_{2}$ & 1 & 1.02 & 2.41 & 2.02 \\
\hline \hline
\end{tabular}

respect to fitting a Gaussian with $\sigma=1$ :

$$
F_{2}=\frac{\sum_{k}\left|A F_{k}^{\prime}\right|}{\sum_{k}\left|A F_{k}^{\prime}\right|_{G}},
$$

where $\left|A F_{k}^{\prime}\right|$ represents the absolute value of the first derivative of the focus measure and $\left|A F_{k}^{\prime}\right|_{G}$ the corresponding value for the fitting Gaussian. Table 1 shows that, again, the Sobel+variance and Tenengrad methods provide the smaller values of $F_{2}$, which correspond also to a better noise tolerance.

In conclusion, the sharpness measure based on Sobel+variance is clearly better than that based on Tenengrad, whereas the smoothness measures are comparable.

\section{Multi-focus visualization techniques}

In this section we present two methods for visualizing the 3D structure of diatoms. The first method is based on image fusion techniques, by combining the most important structures of a specimen from a multifocus image stack into a single fused image. The second approach is based on extracting the $3 \mathrm{D}$ information by deconvolving the multifocus stack.

\subsection{Multifocus fusion}

Image fusion produces a single image from a set of images. In this case, the images to be fused have the same origin, i.e. they are from a multifocus image stack. The main advantages of the fusion process are: (1) the capability of gathering complementary information, and (2) improved reliability by combining redundant information. Since most diatom valves are not flat, a sharp contour and a sharp ornamentation cannot appear together in a single image captured, unless different images taken at different depths are combined. A large number of image fusion techniques has been proposed (e.g. Rockinger, 1999; Wang and Lohmann, 2000). We are only interested in combining multifocus images, but there are also other applications. For example, Seales and Dutta (1996) applied fusion techniques to images taken with inexpensive cameras, with the aim to create focused images in different situations.

Here, we use fusion methods based on simple operations like averaging. The main considerations in the design of the fusion algorithm are its implementation efficiency and portability to other applications, see also Kundur et al. (2000). The most difficult part of the fusion process is the selection of the range of images to be fused. In the case of diatoms we found empirically that it is sufficient to use images in the upper fourth part of the focusing curves, i.e. with values between 0.75 
and 1. These are the images located above the horizontal arrows in Fig. 16, shown in more detail in Fig. 18. After normalizing these images, we apply a binarization using the triangle algorithm, in order to extract the regions that are better focused. This provides a set of binary images that are used to extract the regions that are focused in all images. Each binary image is multiplied with its associated gray level image; hence we can extract the most salient features from each image of the stack (Fig. 20). Figure 18 shows an example of the use of the multifocus fusion technique described here. This figure represents a detailed view of the peak of the focusing curves of Fig. 16, with the eight images to be fused. Figure 19 shows the fused result. This image is computed by averaging the salient features extracted from the image stack, showing a sharp contour and striation. The multifocus fusion algorithm is as follows:

\section{Algorithm 2: Multifocus fusion algorithm}

1. Capture a multifocus image stack

2. Select the range of images to be fused (upper quarter of the focus curve)

3. Background suppression of the selected images, using "rolling ball" method

4. Clip each image between gray levels 0 and 255

5. Threshold each image using the triangle algorithm (Fig. 20)

6. Multiply the binary images of step 5 with the images after step 3

7. Average and normalize the results of step 6.

\subsection{Optical sectioning techniques}

Defocusing in optical microscopy can be modeled as a linear, 3D convolution of the optical density of a specimen with the point spread function of the optics (the microscope lens). There are a number of methods in the literature for inverse filtering or deconvolution, using either measured or estimated optical point spread functions (Preza et al., 1992). It is also possible to estimate the in-focus information-sharp detail and its depth-from the degree of blurring. As for multifocus fusion, a stack of images is taken at equidistant depths, normally 10 to 15 images. Some authors have determined the in-focus depth after applying a highpass filter to each image in the stack, maximizing the result. In this way, a set of focus measures is obtained at each position $(\mathrm{x}, \mathrm{y})$. Then, the depths of surface points at each position are obtained by detecting the peaks (Noguchi and Nayar, 1994). Glasbey (1996) has applied this technique to recover the $3 \mathrm{D}$ valve surfaces of diatoms. Figure 21 shows four images of a diatom taken at different focal planes, and Fig. 22 shows the recovered $3 \mathrm{D}$ surface of the valve, obtained from a total of fourteen images. Such a $3 \mathrm{D}$ reconstruction can be used to create one single image with a sharp contour as well as striation pattern. It can also be used to compute new surface features concerning the $3 \mathrm{D}$ shape of a valve. These features can complement the ones described 


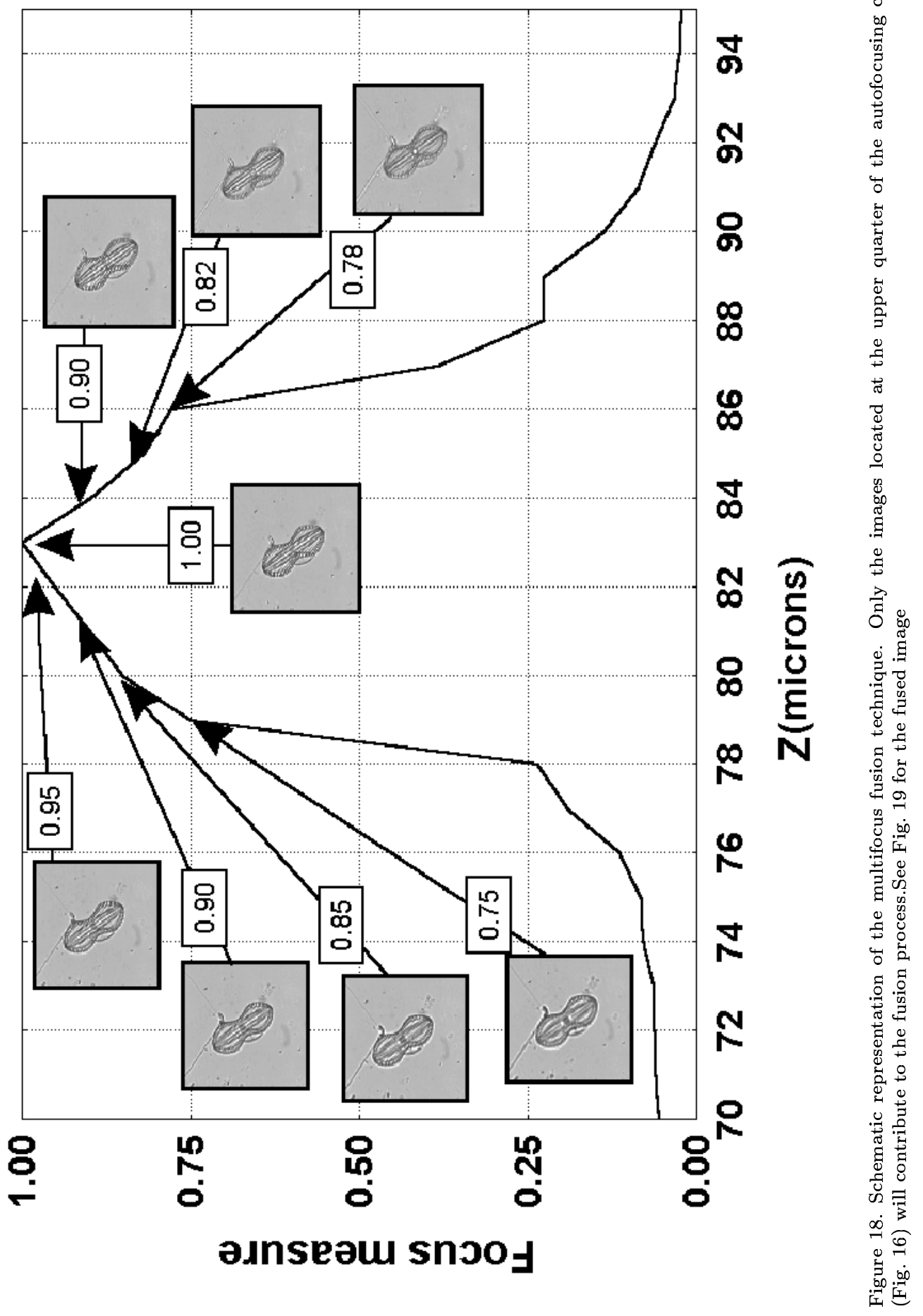




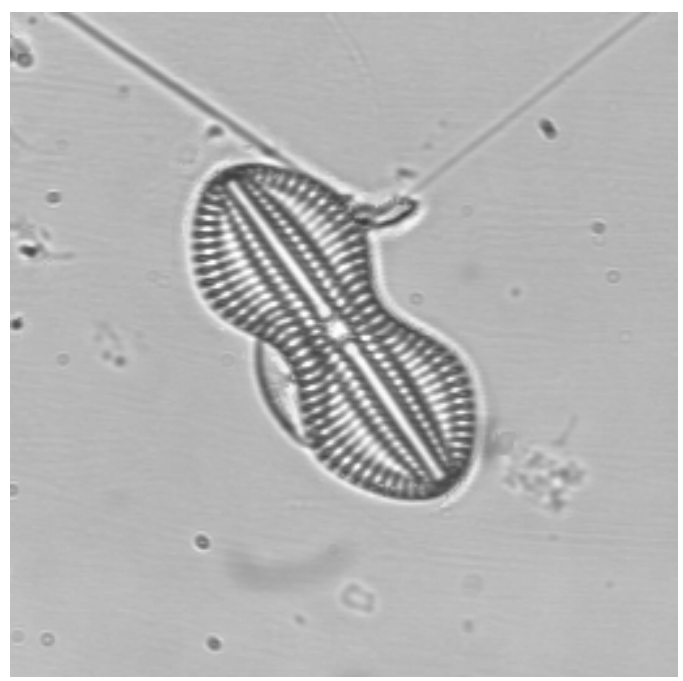

Figure 19. Fused image that results from applying Algorithm 2 to the eight selected images (Fig. 18). The goal is to obtain a single image with a sharp contour as well as striation.
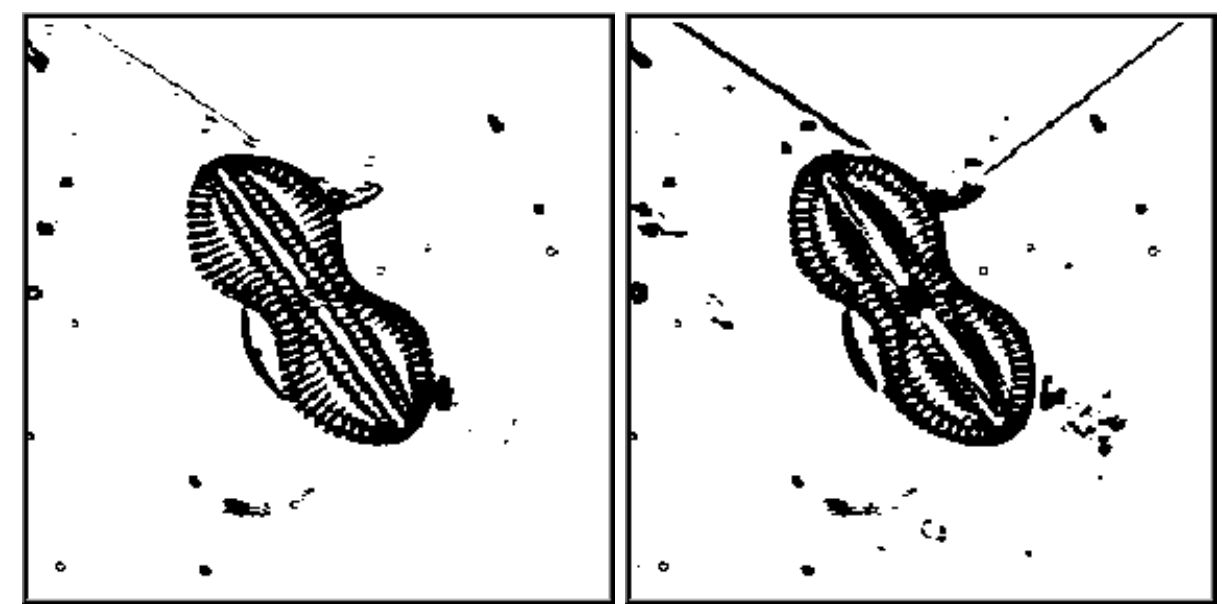

Figure 20. Examples of binary images generated by step 5 of Algorithm 2. Each binary image will be multiplied with its corresponding gray level image in order to extract the focused detail.

in the identification chapters in this book. As far as we know, this information has never been applied by taxonomists, hence this will be studied in the future. 


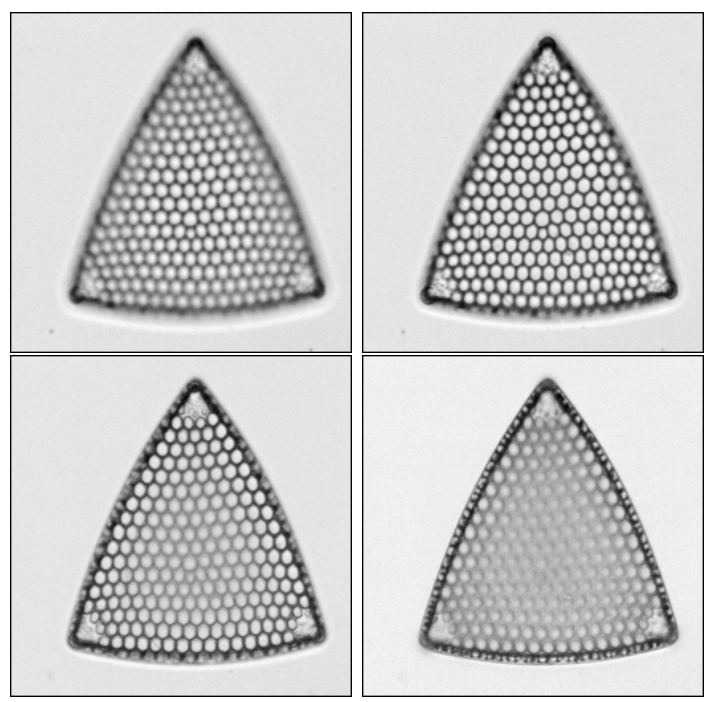

Figure 21. Diatom images taken at different depths. Reprinted with kind permission of Glasbey (1996).

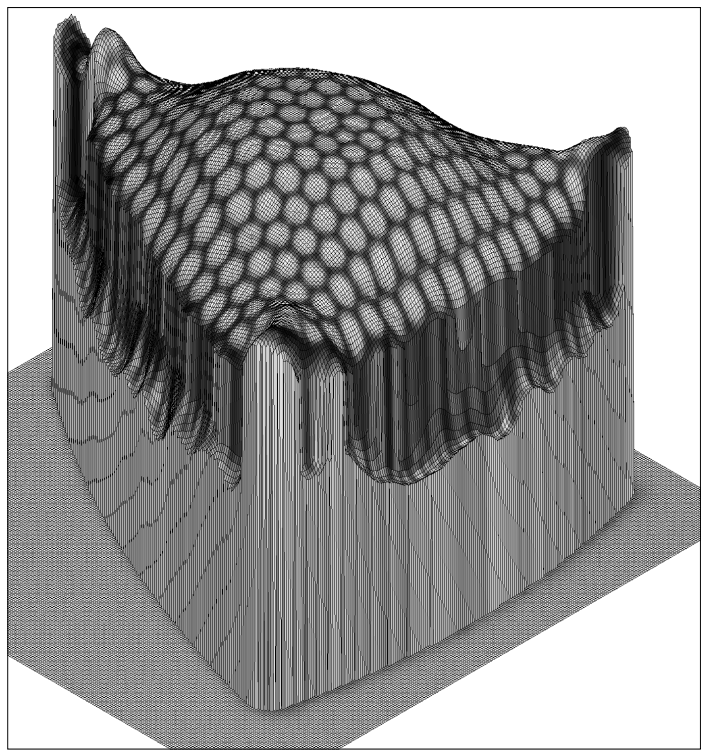

Figure 22. 3D diatom reconstruction from a series of fourteen focal planes. Reprinted with kind permission of Glasbey (1996). 


\section{Conclusions}

In this chapter we have described a robust and efficient slide scanning technique for accurately detecting all candidate particles at low magnification. We have shown two new methods for autofocusing, using a combination of gradient and variance operators. Extensive work has been carried out on autofocusing for a large number of different specimens. The proposed focus measures were compared with a number of techniques from the literature. Significant improvements have been obtained with focusing metrics based on smoothness and peakedness. The particle screening on the basis of phase correlation allows the detection of about $80 \%$ of usable diatom valves. To the best of our knowledge, this is the first study in which all the components required for an automatic slide scanning system have been investigated.

There are several issues that remain to be addressed. The first is to improve the discrimination performance of the particle screening process, together with a systematic statistical analysis, assisted by expert diatomists. The second is to extend the present study to the nanoplankton range. The third concerns an improvement of the multifocus fusion technique. The simple averaging applied can be replaced by a weighted averaging, using principal component analysis of all images to be fused, as suggested by Wang and Lohmann (2000).

\section{References}

Bocker, W., Rolf, W., Muller, W. and Streffer, C. (1996) Investigations about autofocus-algorithms for fluorescent-microscopy. In: SPIE Applications of digital image processing XIX, Vol. 2847, pp. 445-456.

Boddeke, F.R., van Vliet, L.J., Netten, H. and Young, I.T. (1994) Autofocusing in microscopy based on the OTF and sampling. Bioimaging, Vol. 2, pp. 193-203.

Boddeke, F.R. (1998) Quantitative fluorescence microscopy. PhD Thesis, Delft University of Technology, The Netherlands.

Cairns, J., Dickson, K., Lanza, G., Almeida, S. and del Balzo, D. (1972) Coherent optical spatial filtering of diatoms in water pollution monitoring. Archiv Mikrobiologie, Vol. 83, pp. 141-146.

Cairns, J., Dickson, K., Pryfogle, S.P., Almeida, S., Fournier, J.M. and Fuji, H. (1979) Determining the accuracy of coherent optical identification of diatoms. Water Resources Bulletin, Vol. 15, pp. 1770-1775.

Chen, Q. and Defrise, M. (1994) Symmetric phase-only matched filtering of FourierMellin transforms for image registration and recognition. IEEE Trans. Pattern Analysis and Machine Intelligence, Vol. 16, pp. 1156-1168.

Culverhouse, P.F., Simpson, R.G., Ellis, R., Lindley, J.A., Williams, T., Parisini, T., Reguera, B., Bravo, I., Zoppoli, R., Earnshaw, G., McCall, H. and Smith, G. (1996) Automatic classification of field-collected dinoflagellates by artificial neural network. Marine Ecology Progress Series, Vol. 139, pp. 281-287.

da Fontoura, L. and Marcondes, R. (2001) Shape analysis and classification. CRC Press, Boca Raton (FL), USA. 
de Castro, E. and Morandi, C. (1987) Registration of translated and rotated images using finite Fourier transforms. IEEE Trans. Pattern Analysis and Machine Intelligence, Vol. 9, pp. 700-703.

Ellenberger, S.L. and Young, I.T. (1998) Microscope image acquisition. In: Image processing and analysis, R. Baldock and J. Graham (eds), Oxford University Press, Oxford, UK.

Firestone, L.K., Cook, K., Culp, K., Talsania, N. and Preston, K. (1991) Comparison of autofocus methods for automated microscopy. Cytometry, Vol. 12, pp. 195-206.

Glasbey, C.A. (1996) Problems in digital microscopy. Proc. XVIII Int. Biometric Conference, Amsterdam, pp. 183-200.

Groen, F., Young, I.T. and Ligthard, G. (1985) A comparison of different focus functions for use in autofocus algorithms. Cytometry, Vol. 6, pp. 81-91.

Jain, A.K. and Hong, L. (1996) Automatic classification of bacteria culture images. Technical Report, Michigan State University.

Kindratenko, V. (1997) Development and application of image analysis techniques for identification and classification of microscopic particles. PhD Thesis, Universiteit Antwerpen, Belgium.

Krotkov, E. (1987) Focusing. Int. J. Computer Vision, Vol. 1, pp. 223-237.

Kundur, D., Hatzinakos, D. and Leung, H. (2000) Robust classification of blurred imagery. IEEE Trans. Image Processing, Vol. 9, pp. 243-255.

McLaughlin, R.A. (2000) Intelligent algorithms for finding curves and surfaces in real world data. PhD Thesis, Univ. of Western Australia, Dept. Computer and Electronic Engineering.

Nayar, S.K. and Nakagawa, Y. (1994) Shape from focus. IEEE Trans. Pattern Analysis and Machine Intelligence, Vol. 16, pp. 824-831.

Neveu, C. (1999) Formal models of blur detection. Perception, Supplement, Vol. 28, p. 147.

Noguchi, M. and Nayar, S.K. (1994) Microscopic shape from focus using active illumination. Proc. Int. Conf. Pattern Recognition, IEEE Computer Society, Los Alamitos (CA), USA, pp. 147-152.

Pech-Pacheco, J.L. and Alvarez-Borrego, J. (1998) Optical-digital system applied to the identification of five phytoplankton species. Marine Biology, Vol. 132, pp. 357-365.

Pech-Pacheco, J.L., Alvarez-Borrego, J., Orellana-Cepeda, E. and Cortés-Altamirano, R. (1999) Diffraction patterns applicability in identification of Ceratium species. J. Plankton Research, accepted for publication.

Pech-Pacheco, J.L. and Cristóbal, G. (2000) Diatom autofocusing in brightfield microscopy: a comparative study. Proc. Int. Conf. on Pattern Recognition, Barcelona, IEEE Computer Soc., Los Alamitos (CA), USA, Vol. 3, pp. 318-321.

Pech-Pacheco, J.L., Cristóbal, G., Alvarez-Borrego, J. and Cohen, L. (2000) Power cepstral image analysis through the scale transform. Proc. SPIE, Vol. 4113, pp. 68-79.

Pech-Pacheco, J.L. and Cristóbal, G. (2001) Automatic system for phytoplanktonic algae identification. Limnetica, submitted. 
Preza, C., Miller, M.I., Lewis, J.T. and McNally, J.G. (1992) Regularized linear method for reconstruction of three-dimensional microscopic objects from optical sections. J. Optical Society of America A, Vol. 9, pp. 219-228.

Rockinger, O. (1999) Multiresolution-Verfahren zur Fusion dynamischer Bildfolgen. PhD Thesis, Technische Universität Berlin, Germany.

Russ, J.C. (1995) The image processing handbook. CRC press, Boca Raton (FA), USA.

Schlag, J.F., Sanderson, A.C., Neuman, C.P., Wimberly, F.C. (1983) Implementation of automatic focusing algorithms for a computer vision system with camera control. Technical Report CMU-RI-TR-83-14, Robotics Institute, Carnegie Mellon University.

Seales, W.B. and Dutta, S. (1996) Everywhere in focus image fusion using controllable cameras. Proc. SPIE, Vol. 2905, pp. 227-234.

Seul, M., O'Gorman, L. and Sammon, M.J. (2000) Practical algorithms for image analysis. Description, examples and code. Cambridge University Press, Cambridge, UK.

Sternberg, S.R. (1983) Biomedical image processing. Computer Magazine, Vol. 16, pp. 22-34.

Subbarao, M., Choi, T. and Nikzad, A. (1993) Focusing techniques. Optical Engineering, Vol. 32, pp. 2824-2836.

Sun, C. (1995) Symmetry detection using gradient information. Pattern Recognition Letters, Vol. 16, pp. 987-996.

Tenenbaum, J.M. (1970) Accomodation in computer vision, $\mathrm{PhD}$ Dissertation, Stanford University, USA.

Vollath, D. (1987) Automatic focusing by autocorrelative methods. J. Microscopy, Vol. 147, pp. 279-288.

Wang, Y. and Lohmann, B. (2000) Multisensor image fusion: concept, method and applications. Technical Report, Inst. of Automation Technology, Univ. Bremen, Germany.

Yeo, T., Ong, S., Jayasooriah and Sinniah, R. (1993) Autofocusing for tissue microscopy. Image and Vision Computing, Vol. 11, pp. 629-639.

Young, I.T., Gerbrands, J.J. and van Vliet, L.J. (1998) Image processing fundamentals. In: The digital signal processing handbook, V.K. Madisetti and D.B. Williams (eds), CRC Press, Boca Raton (FA), USA.

Zack, G.W., Rogers, W.E. and Latt, S.A. (1977) Automatic measurement of sister chromatid exchange frequency. J. Histochemistry and Cytochemistry, Vol. 25, pp. 741-753. 